\title{
Rehabilitation Nursing
}

National Cancer Institute

\section{Source}

National Cancer Institute. Rehabilitation Nursing. NCI Thesaurus. Code C17959.

Rehabilitation nursing is a specialty devoted to assisting individuals of all ages and cultural backgrounds to accomplish the tasks of daily living to their greatest potential and return to their premorbid lifestyle. (ARN) 